\title{
An Improvement of Global Error Bound for the Generalized Nonlinear Complementarity Problem over a Polyhedral Cone
}

\author{
Hongchun Sun, ${ }^{1}$ Yiju Wang, ${ }^{2}$ Houchun $\mathrm{Zhou}^{1}$ and Shengjie $\mathrm{Li}^{3}$ \\ ${ }^{1}$ School of Sciences, Linyi University, Linyi, Shandong 276005, China \\ ${ }^{2}$ School of Management Science, Qufu Normal University, Rizhao, Shandong 276800, China \\ ${ }^{3}$ College of Mathematics and Statistics, Chongqing University, Chongqing 401331, China
}

Correspondence should be addressed to Yiju Wang; wyiju@hotmail.com

Received 27 February 2014; Accepted 16 April 2014; Published 29 April 2014

Academic Editor: Fu-quan Xia

Copyright (C) 2014 Hongchun Sun et al. This is an open access article distributed under the Creative Commons Attribution License, which permits unrestricted use, distribution, and reproduction in any medium, provided the original work is properly cited.

We consider the global error bound for the generalized nonlinear complementarity problem over a polyhedral cone (GNCP). By a new technique, we establish an easier computed global error bound for the GNCP under weaker conditions, which improves the result obtained by Sun and Wang (2013) for GNCP.

\section{Introduction}

Let $\mathscr{K}=\left\{v \in R^{m} \mid A v \geq 0, B v=0\right\}$ be a polyhedral cone in $R^{m}$ for matrices $A \in R^{s \times m}, B \in R^{t \times m}$, and let $\mathscr{K}^{\circ}$ be its dual cone; that is,

$$
\mathscr{K}^{\circ}=\left\{u \in R^{m} \mid u=A^{\top} \lambda_{1}+B^{\top} \lambda_{2}, \lambda_{1} \in R_{+}^{s}, \lambda_{2} \in R^{t}\right\} .
$$

For continuous mappings, $F, G: R^{n} \rightarrow R^{m}$, the generalized nonlinear complementarity problem, abbreviated as GNCP, is to find vector $x^{*} \in R^{n}$ such that

$$
F\left(x^{*}\right) \in \mathscr{K}, \quad G\left(x^{*}\right) \in \mathscr{K}^{\circ}, \quad F\left(x^{*}\right)^{\top} G\left(x^{*}\right)=0 .
$$

Throughout this paper, the solution set of the GNCP, denoted by $X^{*}$, is assumed to be nonempty.

The GNCP is a direct generalization of the classical nonlinear complementarity problem and a special case of the general variational inequalities problem [1]. The GNCP was deeply discussed [2-7] after the work in [8]. It is well known that the global error bound is an important tool for theoretical analysis and numerical treatment for a mathematical problem $[9,10]$. The global error bound estimation for GNCP with the mapping being $\gamma$-strongly $G$-monotone was discussed in [4], a global error bound estimation for GNCP with the mapping being $\gamma$-strongly monotone and Hölder continuous was established in [5], and a global error bound for the GNCP for the linear and monotonic case was also established in $[6,7]$.

This paper is a follow-up to $[4,5,11]$, as in these papers we will establish the global error bound estimation of the GNCP under weaker conditions than that needed in $[4,5,11]$. Based on a new technique, we establish a global error bound for the GNCP in terms of an easier computed residual function. The results obtained in this paper can be taken as an improvement of the existing results for GNCP and variational inequalities problem $[4,5,11-13]$.

To end this section, we give some notations used in this paper. Vectors considered in this paper are taken in Euclidean space $R^{n}$ equipped with the usual inner product, and the Euclidean 2-norm and 1-norm of vector in $R^{n}$ are, respectively, denoted by $\|\cdot\|$ and $\|\cdot\|_{1}$. We use $R_{+}^{n}$ to denote the nonnegative orthant in $R^{n}$ and use $x_{+}$and $x_{-}$to denote the vectors composed by elements $\left(x_{+}\right)_{i}:=\max \left\{x_{i}, 0\right\},\left(x_{-}\right)_{i}:=$ $\max \left\{-x_{i}, 0\right\}$ and $1 \leq i \leq n$, respectively. For simplicity, we use $(x ; y)$ to denote vector $\left(x^{\top}, y^{\top}\right)^{\top}$, use $I$ to denote the identity matrix with appropriate dimension, use $x \geq 0$ to denote a nonnegative vector $x \in R^{n}$, and use $\operatorname{dist}\left(x, X^{*}\right)$ to denote the distance from point $x$ to the solution set $X^{*}$.

\section{Global Error Bound for the GNCP}

First, we give the following definition used in the subsequent. 
Definition 1. The mapping $F: R^{n} \rightarrow R^{m}$ is said to be $\gamma$ uniform $p$-function with respect to $G: R^{n} \rightarrow R^{m}$ if there are constants $c_{1}>0$ and $\gamma>0$ such that

$$
\begin{gathered}
\max _{1 \leq i \leq m}\left\{[F(x)-F(y)]_{i}[G(x)-G(y)]_{i}\right\} \\
\geq c_{1}\|G(x)-G(y)\|^{1+\gamma}, \\
\forall x, y \in R^{n} .
\end{gathered}
$$

Remark 2. Based on this definition, $\gamma$-uniform $p$-function with respect to $G$ is weaker than $\gamma$-strongly $G$-monotonicity by Definition 1 in [4], and if

$$
F(x)=M x+p, \quad G(x)=N x+q
$$

with $M, N \in R^{m \times n}, p, q \in R^{m}$, then the above definition is equivalent in which the matrix $M^{\top} N$ is a $p$-matrix [14]. For example, let

$$
M=\left(\begin{array}{cc}
1 & -4 \\
1 & 1
\end{array}\right), \quad N=\left(\begin{array}{ll}
1 & 0 \\
0 & 1
\end{array}\right), \quad p=q=\left(\begin{array}{l}
0 \\
0
\end{array}\right) .
$$

By Theorem 2.1.15 in [14], it is easy to verify that $M^{\top} N$ is a $p$ matrix. However, letting $x=(1 ; 2)$, we note that $x^{\top} M^{\top} N x=$ $-1<0$ which shows that $M^{\top} N$ is not positive definite; that is, $F$ is not strongly monotonicity with respect to mapping $G$.

Now, we give some assumptions for our analysis based on Definition 1.

Assumption 3. For mappings $F, G$ and matrix $A$ involved in the GNCP, we assume that

(A1) mapping $F$ is $\gamma$-uniform $p$-function with respect to mapping $G$;

(A2) matrix $A^{\top}$ has full-column rank.

In the following, we give the conclusion established in [2].

Theorem 4. A point $x^{*} \in R^{n}$ is a solution of the GNCP if and only if there exist $\lambda_{1}^{*} \in R^{s}$ and $\lambda_{2}^{*} \in R^{t}$, such that

$$
\begin{gathered}
A F\left(x^{*}\right) \geq 0, \\
B F\left(x^{*}\right)=0, \\
\lambda_{1}^{*} \geq 0, \\
\left(F\left(x^{*}\right)\right)^{\top} G\left(x^{*}\right)=0, \\
G\left(x^{*}\right)=A^{\top} \lambda_{1}^{*}+B^{\top} \lambda_{2}^{*} .
\end{gathered}
$$

From Theorem 4, under Assumption 3 (A2), similar to discussion in [4], we can transform the system (6) into the following system in which neither $\lambda_{1}$ nor $\lambda_{2}$ is involved:

$$
\begin{gathered}
A F(x) \geq 0, \\
B F(x)=0, \\
(F(x))^{\top} G(x)=0, \\
U G(x) \geq 0, \\
V G(x)=0,
\end{gathered}
$$

where

$$
\begin{gathered}
U=\left\{-A_{L}^{-1} B^{\top}\left[\left(A^{\top} A_{L}^{-1}-I\right) B^{\top}\right]^{+}\left[A^{\top} A_{L}^{-1}-I\right]+A_{L}^{-1}\right\}, \\
V=\left\{A^{\top}\left\{-A_{L}^{-1} B^{\top}\left[\left(A^{\top} A_{L}^{-1}-I\right) B^{\top}\right]^{+}\left[A^{\top} A_{L}^{-1}-I\right]+A_{L}^{-1}\right\}\right. \\
\left.+B^{\top}\left[\left(A^{\top} A_{L}^{-1}-I\right) B^{\top}\right]^{+}\left[A^{\top} A_{L}^{-1}-I\right]-I\right\} .
\end{gathered}
$$

For the ease of description, let $\mu=F(x)$ and $\nu=G(x)$. Then, system (7) can be written as

$$
\begin{aligned}
& A \mu \geq 0, \\
& B \mu=0, \\
& \mu^{\top} v=0, \\
& U \nu \geq 0, \\
& V \nu=0,
\end{aligned}
$$

where the solution set of (9) is denoted by $\Omega^{*}$.

In the following, we give the error bound for a single quadratic function to reach our aims.

Lemma 5. Let $S_{1}:=\left\{\omega \in R^{2 m} \mid f(\omega)=0\right\}$. Then, one has

$$
\operatorname{dist}\left(\omega, S_{1}\right) \leq \tau\|f(\omega)\|^{1 / 2}
$$

where $\tau>0$ is a constant, $\omega=(\mu ; \nu)$, and $f(\omega)=\mu^{\top} v$.

Proof. For any $\omega \in R^{2 m}$, let $\mu=\left(\mu_{1}, \mu_{2}, \ldots, \mu_{m}\right)^{\top}, \nu=$ $\left(v_{1}, v_{2}, \ldots, v_{m}\right)^{\top}$, and

$$
\omega_{i}= \begin{cases}\mu_{i} & 1 \leq i \leq m \\ \nu_{i} & m+1 \leq i \leq 2 m\end{cases}
$$

Set $\mu_{i}=\xi_{i}+\xi_{\underline{m}+i}, \nu_{i}=\xi_{i}-\xi_{m+i}, i=1,2, \ldots, m$, and $\xi=$ $\left(\xi_{1}, \xi_{2}, \ldots, \xi_{2 m}\right)^{\top}$. Obviously, this linear transformation is an invertible; that is, there exists an invertible matrix $P \in R^{2 m \times 2 m}$ such that $\omega=P \xi$, and one has

$$
f(\omega)=\mu^{\top} \nu=\sum_{i=1}^{m} \mu_{i} \nu_{i}=\sum_{i=1}^{m} \xi_{i}^{2}-\sum_{i=m+1}^{2 m} \xi_{i}^{2}=: g(\xi) .
$$

Without loss of generality, we assume $f(\omega)>0$. Define

$$
\begin{gathered}
\theta=\left(\frac{\sum_{i=m+1}^{2 m} \xi_{i}^{2}}{g(\xi)+\sum_{i=m+1}^{2 m} \xi_{i}^{2}}\right)^{1 / 2}=\left(\frac{\sum_{i=m+1}^{2 m} \xi_{i}^{2}}{\sum_{i=1}^{m} \xi_{i}^{2}}\right)^{1 / 2}, \\
\bar{\xi}_{i}= \begin{cases}\theta \xi_{i} & 1 \leq i \leq m, \\
\xi_{i} & m+1 \leq i \leq 2 m .\end{cases}
\end{gathered}
$$


It is easy to verify that $0 \leq \theta \leq 1$ and

$$
\begin{aligned}
g(\bar{\xi}) & =\theta^{2} \sum_{i=1}^{m} \xi_{i}^{2}-\sum_{i=m+1}^{2 m} \xi_{i}^{2} \\
& =\left(\frac{\sum_{i=m+1}^{2 m} \xi_{i}^{2}}{\sum_{i=1}^{m} \xi_{i}^{2}}\right) \sum_{i=1}^{m} \xi_{i}^{2}-\sum_{i=m+1}^{2 m} \xi_{i}^{2} \\
& =\sum_{i=m+1}^{2 m} \xi_{i}^{2}-\sum_{i=m+1}^{2 m} \xi_{i}^{2} \\
& =0 .
\end{aligned}
$$

Let $\bar{\omega}=P \bar{\xi}$, and one has $f(\bar{\omega})=g(\bar{\xi})=0$. Therefore, $\bar{\omega} \in S_{1}$. Moreover, one has

$$
\begin{aligned}
& \|\omega-\bar{\omega}\| \\
& =\|P \xi-P \bar{\xi}\| \\
& \leq\|P\|\|\xi-\bar{\xi}\| \\
& =\|P\|\left(\sum_{i=1}^{2 m}\left(\xi_{i}-\bar{\xi}_{i}\right)^{2}\right)^{1 / 2} \\
& =\|P\|\left(\sum_{i=1}^{m}\left(\xi_{i}-\theta \xi_{i}\right)^{2}\right)^{1 / 2} \\
& =\|P\|(1-\theta)\left(\sum_{i=1}^{m} \xi_{i}^{2}\right)^{1 / 2} \\
& =\|P\| \frac{\left(1-\theta^{2}\right)}{1+\theta}\left(\sum_{i=1}^{m} \xi_{i}^{2}\right)^{1 / 2} \\
& =\frac{\|P\|}{1+\theta}\left(1-\frac{\sum_{i=m+1}^{2 m} \xi_{i}^{2}}{g(\xi)+\sum_{i=m+1}^{2 m} \xi_{i}^{2}}\right)\left(\sum_{i=1}^{m} \xi_{i}^{2}\right)^{1 / 2} \\
& =\frac{\|P\| g(\xi)}{(1+\theta)\left(\sum_{i=1}^{m} \xi_{i}^{2}\right)}\left(\sum_{i=1}^{m} \xi_{i}^{2}\right)^{1 / 2} \\
& =\frac{\|P\| g(\xi)}{(1+\theta)\left(\sum_{i=1}^{m} \xi_{i}^{2}\right)^{1 / 2}} \\
& =\frac{\|P\| g(\xi)}{\left(\sum_{i=1}^{m} \xi_{i}^{2}\right)^{1 / 2}+\theta\left(\sum_{i=1}^{m} \xi_{i}^{2}\right)^{1 / 2}} \\
& =\frac{\|P\| g(\xi)}{\left(\sum_{i=1}^{m} \xi_{i}^{2}\right)^{1 / 2}+\left(\left(\sum_{i=m+1}^{2 m} \xi_{i}^{2}\right) /\left(\sum_{i=1}^{m} \xi_{i}^{2}\right)\right)^{1 / 2}\left(\sum_{i=1}^{m} \xi_{i}^{2}\right)^{1 / 2}} \\
& =\frac{\|P\| g(\xi)}{\left(\sum_{i=1}^{m} \xi_{i}^{2}\right)^{1 / 2}+\left(\sum_{i=m+1}^{2 m} \xi_{i}^{2}\right)^{1 / 2}}
\end{aligned}
$$

$$
\begin{aligned}
& \leq \frac{\|P\| g(\xi)}{\left(\sum_{i=1}^{m} \xi_{i}^{2}+\sum_{i=m+1}^{2 m} \xi_{i}^{2}\right)^{1 / 2}} \\
& \leq \frac{\|P\| g(\xi)}{g(\xi)^{1 / 2}}=\|P\| g(\xi)^{1 / 2}=\|P\| f(\omega)^{1 / 2},
\end{aligned}
$$

where the third equality follows from the definition of $\bar{\xi}$, the sixth and tenth equations are due to the definition of $\theta$, respectively, the second inequality follows from the fact that

$$
a^{1 / 2}+b^{1 / 2} \geq(a+b)^{1 / 2}, \quad \forall a, b \in R_{+},
$$

and the third inequality follows from the fact that

$$
g(\xi) \leq \sum_{i=1}^{m} \xi_{i}^{2}+\sum_{i=m+1}^{2 m} \xi_{i}^{2} .
$$

And, letting $\tau=\|P\|$, then the desired result follows.

To establish a global error bound for GNCP, we also give the following result from [15] on the error bound for a polyhedral cone.

Lemma 6. For polyhedral cone $P=\left\{x \in R^{n} \mid D_{1} x=\right.$ $\left.d_{1}, B_{1} x \leq b_{1}\right\}$ with $D_{1} \in R^{l \times n}, B_{1} \in R^{m \times n}, d_{1} \in R^{l}$, and $b_{1} \in R^{m}$, there exists a constant $c_{2}>0$ such that

$$
\operatorname{dist}(x, P) \leq c_{2}\left[\left\|D_{1} x-d_{1}\right\|+\left\|\left(B_{1} x-b_{1}\right)_{+}\right\|\right], \quad \forall x \in R^{n} .
$$

Before proceeding, we present the following definition introduced in [16] with constant $\delta=1$.

Definition 7. The mapping $G: R^{n} \rightarrow R^{m}$ is said to be $\delta$ strongly nonexpanding with a constant $\alpha>0$, if $\| G(x)-$ $G(y)\|\geq \alpha\| x-y \|^{\delta}$, where $\delta>0$.

Now, we are at the position to state our main results in this paper.

Theorem 8. Suppose that $F$ is $\gamma$-uniform p-function with respect to mapping $G$ with positive constants $c_{1}$ and $\gamma$, respectively, and $G$ is $\delta$-strongly nonexpanding with positive constants $\alpha$ and $\delta$, respectively. Then there exists constant $\rho_{1}>0$ such that

$$
\begin{aligned}
\operatorname{dist}\left(x, X^{*}\right) \leq \rho_{1}\{ & \left\|[A F(x)]_{-}\right\|+\|B F(x)\| \\
& +\left\|[U G(x)]_{-}\right\|+\|V G(x)\| \\
& \left.+\left\|\left[F(x)^{\top} G(x)\right]\right\|^{1 / 2}\right\}^{2 /(1+\gamma) \delta}, \quad \forall x \in R^{n} .
\end{aligned}
$$

Proof. Using Lemma 5, for any $\omega \in R^{2 m}$, there exists $\bar{\omega} \in S_{1}$ such that

$$
\|\omega-\bar{\omega}\| \leq \tau\|[f(\omega)]\|^{1 / 2}
$$


where $S_{1}$ is defined in Lemma 5. Let

$$
\begin{gathered}
\Omega=\left\{\omega \in R^{2 m} \mid A(I, 0) \omega \geq 0, B(I, 0) \omega=0,\right. \\
U(0, I) \omega \geq 0, V(0, I) \omega=0\} .
\end{gathered}
$$

From (9), we have $\Omega^{*}=\Omega \bigcap S_{1}$. For convenience, we also let

$$
\begin{array}{r}
\Psi(\omega)=(-A(I, 0) \omega,-B(I, 0) \omega,-U(0, I) \omega \\
-V(0, I) \omega, B(I, 0) \omega, V(0, I) \omega)_{+}
\end{array}
$$

Using Lemma 6, for any $\omega \in S_{1}$, there exists $\omega^{*} \in \Omega^{*}$ such that

$$
\begin{gathered}
\left\|\omega-\omega^{*}\right\| \leq c_{3}\left[\left\|(-A(I, 0) \omega)_{+}\right\|+\left\|(-U(0, I) \omega)_{+}\right\|\right. \\
+\|B(I, 0) \omega\|+\|V(0, I) \omega\|] \\
\leq c_{3}\left[\left\|(-A(I, 0) \omega)_{+}\right\|+\left\|(-U(0, I) \omega)_{+}\right\|\right. \\
+\left\|(B(I, 0) \omega)_{+}\right\| \\
+\left\|(-B(I, 0) \omega)_{+}\right\|+\left\|(V(0, I) \omega)_{+}\right\| \\
\left.+\left\|(-V(0, I) \omega)_{+}\right\|\right] \\
\leq c_{3}\left\{\left\|(-A(I, 0) \omega)_{+}\right\|_{1}+\left\|(-U(0, I) \omega)_{+}\right\|_{1}\right. \\
+\left\|(B(I, 0) \omega)_{+}\right\|_{1} \\
+\left\|(-B(I, 0) \omega)_{+}\right\|_{1}+\left\|(V(0, I) \omega)_{+}\right\|_{1} \\
\left.\quad+\left\|(-V(0, I) \omega)_{+}\right\| \|_{1}\right\} \\
=c_{3}\|\Psi(\omega)\|_{1} \\
\leq c_{3} \sqrt{2 s+2 t+2 m\|\Psi(\omega)\|}
\end{gathered}
$$

where $c_{3}$ is a positive constant and the third and fourth inequalities follow from the fact that $\|x\| \leq\|x\|_{1} \leq \sqrt{n}\|x\|$, for all $x \in R^{n}$.

Furthermore,

$$
\begin{aligned}
& \|\Psi(\omega)-\Psi(\bar{\omega})\| \\
& =\|(-A(I, 0) \omega,-B(I, 0) \omega,-U(0, I) \omega \\
& -V(0, I) \omega, B(I, 0) \omega, V(0, I) \omega)_{+} \\
& -(-A(I, 0) \bar{\omega},-B(I, 0) \bar{\omega},-U(0, I) \bar{\omega} \\
& -V(0, I) \bar{\omega}, B(I, 0) \bar{\omega}, V(0, I) \bar{\omega})_{+} \| \\
& \left.\left.=\| P_{R_{+}^{2 s+2 t+2 m}\{(-A(I, 0) \omega,-B(I, 0) \omega,-U(0, I) \omega,} \quad-V(0, I) \omega, B(I, 0) \omega, V(0, I) \omega\right)\right\} \\
& -P_{R_{+}^{2 s+2 t+2 m}}\{(-A(I, 0) \bar{\omega},-B(I, 0) \bar{\omega},-U(0, I) \bar{\omega} \\
& \quad-V(0, I) \bar{\omega}, B(I, 0) \bar{\omega}, V(0, I) \bar{\omega})\} \|
\end{aligned}
$$

$$
\begin{aligned}
\leq & \|\{(-A(I, 0) \omega,-B(I, 0) \omega,-U(0, I) \omega, \\
& -V(0, I) \omega, B(I, 0) \omega, V(0, I) \omega)\} \\
- & \{(-A(I, 0) \bar{\omega},-B(I, 0) \bar{\omega},-U(0, I) \bar{\omega}, \\
& \quad-V(0, I) \bar{\omega}, B(I, 0) \bar{\omega}, V(0, I) \bar{\omega})\} \| \\
\leq & \|A(I, 0) \omega-A(I, 0) \bar{\omega}\|+2\|B(I, 0) \omega-B(I, 0) \bar{\omega}\| \\
& +\|U(0, I) \omega-U(0, I) \bar{\omega}\|+2\|V(0, I) \omega-V(0, I) \bar{\omega}\| \\
\leq & \|A(I, 0)\|+2\|B(I, 0)\|+\|U(0, I)\| \\
& +2\|V(0, I)\|)\|\omega-\bar{\omega}\|,
\end{aligned}
$$

where the second equality follows from the fact that

$$
\min \{a, b\}=a-P_{R_{+}}(a-b), \quad \forall a, b \in R,
$$

and the first inequality is by nonexpanding property of projection operator. Combining (24), one has

$$
\begin{aligned}
\|\Psi(\bar{\omega})\| \leq & \|\Psi(\omega)\| \\
+ & (\|A(I, 0)\|+2\|B(I, 0)\| \\
& +\|U(0, I)\|+2\|V(0, I)\|)\|\omega-\bar{\omega}\| .
\end{aligned}
$$

Combining (23) with (26), for any $\omega \in R^{2 m}$, we have

$$
\begin{aligned}
& \left\|\omega-\omega^{*}\right\| \leq\|\omega-\bar{\omega}\|+\left\|\bar{\omega}-\omega^{*}\right\| \\
& \leq\|\omega-\bar{\omega}\|+\sigma\|\Psi(\bar{\omega})\| \\
& \leq\|\omega-\bar{\omega}\| \\
& +\sigma(\|\Psi(\omega)\|+(\|A(I, 0)\|+2\|B(I, 0)\| \\
& +\|U(0, I)\| \\
& +2\|V(0, I)\|)\|\omega-\bar{\omega}\|) \\
& \leq \sigma\|\Psi(\omega)\| \\
& +[\sigma(\|A(I, 0)\|+2\|B(I, 0)\| \\
& +\|U(0, I)\|+2\|V(0, I)\|)+1]\|\omega-\bar{\omega}\| \\
& \leq \sigma\|\Psi(\omega)\| \\
& +[\sigma(\|A(I, 0)\|+2\|B(I, 0)\|+\|U(0, I)\| \\
& +2\|V(0, I)\|)+1] \tau\|[f(\omega)]\|^{1 / 2} \\
& \leq \eta\left(\|\Psi(\omega)\|+\|[f(\omega)]\|^{1 / 2}\right) \\
& \leq \eta\left(\|\Psi(\omega)\|_{1}+\|[f(\omega)]\|^{1 / 2}\right) \\
& \leq \eta\left(\left\|(-A(I, 0) \omega)_{+}\right\|_{1}+\left\|(-U(0, I) \omega)_{+}\right\|_{1}\right. \\
& +\|B(I, 0) \omega\|_{1} \\
& \left.+\|V(0, I) \omega\|_{1}+\|[f(\omega)]\|^{1 / 2}\right)
\end{aligned}
$$




$$
\begin{aligned}
& \leq \eta(\sqrt{s}\left\|(-A(I, 0) \omega)_{+}\right\|+\sqrt{s}\left\|(-U(0, I) \omega)_{+}\right\| \\
&+\sqrt{t}\|B(I, 0) \omega\| \\
&\left.+\sqrt{m}\|V(0, I) \omega\|+\|[f(\omega)]\|^{1 / 2}\right) \\
& \leq c_{4}\left(\left\|(-A(I, 0) \omega)_{+}\right\|+\left\|(-U(0, I) \omega)_{+}\right\|\right. \\
&+\|B(I, 0) \omega\| \\
&\left.+\|V(0, I) \omega\|+\|[f(\omega)]\|^{1 / 2}\right),
\end{aligned}
$$

where the second inequality follows from (23) with constants $\sigma=c_{3} \sqrt{2 s+2 t+2 m}$ and $\omega=\bar{\omega}$, the third inequality uses (26), the fifth inequality follows from (20), the sixth inequality follows from the fact that

$$
\begin{aligned}
\eta=\max \{\sigma,[\sigma & (\|A(I, 0)\|+2\|B(I, 0)\| \\
& +\|U(0, I)\|+2\|V(0, I)\|)+1] \tau\},
\end{aligned}
$$

the seventh and ninth inequalities follow from the fact that

$$
\|x\| \leq\|x\|_{1} \leq \sqrt{n}\|x\|, \quad \forall x \in R^{n},
$$

and the last inequality follows by letting $c_{4}=\eta \max \{\sqrt{s}$, $\sqrt{t}, \sqrt{m}, 1\}$.

For any $x \in R^{n}$, let $\omega=(\mu, \nu)=(F(x), G(x)) \in R^{2 m}$. Then there exists $\omega^{*}=\left(\mu^{*}, \nu^{*}\right)=\left(F\left(x^{*}\right), G\left(x^{*}\right)\right) \in \Omega^{*}$ such that

$$
\begin{aligned}
& \text { dist }^{(1+\gamma) \delta}\left(x, X^{*}\right) \\
& \quad \leq\left\|x-x^{*}\right\|^{(1+\gamma) \delta} \\
& \quad \leq \frac{1}{\alpha^{1+\gamma}}\left\|G(x)-G\left(x^{*}\right)\right\|^{1+\gamma} \\
& \quad \leq \frac{1}{c_{1} \alpha^{1+\gamma}} \max _{1 \leq i \leq n}\left\{[F(x)-F(y)]_{i}[G(x)-G(y)]_{i}\right\} \\
& \quad \leq \frac{1}{c_{1} \alpha^{1+\gamma}}\left\|F(x)-F\left(x^{*}\right)\right\|\left\|G(x)-G\left(x^{*}\right)\right\| \\
& \quad \leq \frac{1}{2 c_{1} \alpha^{1+\gamma}}\left\{\left\|F(x)-F\left(x^{*}\right)\right\|^{2}+\left\|G(x)-G\left(x^{*}\right)\right\|^{2}\right\} \\
& =\frac{1}{2 c_{1} \alpha^{1+\gamma}}\left\|\omega-\omega^{*}\right\|^{2} \\
& \leq \frac{1}{2 c_{1} \alpha^{1+\gamma}} c_{4}^{2}\left(\left\|(-A(I, 0) \omega)_{+}\right\|+\left\|(-U(0, I) \omega)_{+}\right\|\right. \\
& \quad+\|B(I, 0) \omega\|
\end{aligned}
$$

$$
\begin{gathered}
\leq \frac{1}{2 c_{1} \alpha^{1+\gamma}} c_{4}^{2}\left(\left\|(A(I, 0) \omega)_{-}\right\|+\left\|(U(0, I) \omega)_{-}\right\|\right. \\
+\|B(I, 0) \omega\| \\
\left.+\|V(0, I) \omega\|+\|[f(\omega)]\|^{1 / 2}\right)^{2} \\
\leq \frac{1}{2 c_{1} \alpha^{1+\gamma}} c_{4}^{2}\left\{\left\|[A \mu]_{-}\right\|+\|B \mu\|+\left\|[U \nu]_{-}\right\|+\|V \nu\|\right. \\
\left.+\left\|\mu^{\top} \nu\right\|^{1 / 2}\right\}^{2},
\end{gathered}
$$

where the second inequality follows from Definition 7 with constant $\alpha>0$, the third inequality follows from Definition 1 with constants $c_{1}>0$ and $\gamma>0$, the fifth inequality follows from the fact that $a^{2}+b^{2} \geq 2 a b$, for all $a, b \in R$, and the sixth inequality is by (27). By (30) and letting $\rho_{1}=$ $\left\{\left(1 /\left(2 c_{1} \alpha^{1+\gamma}\right)\right) c_{4}^{2}\right\}^{1 /(1+\gamma) \delta}$, then the desired result follows.

Remark 9. Firstly, from remark of Definition 1 , the conditions that $F$ is $\gamma$-uniform $p$-function with respect to mapping $G$ and $G$ is $\delta$-strongly nonexpanding in Theorem 8 are weaker than the conditions that $F$ is $\gamma$-strongly $G$-monotone and $G$ is strongly nonexpanding (i.e., $\delta=1$ ) in Theorem 13 in [4]. In addition, the result in Theorem 8 is stronger than that in Theorem 13 in [4]. Thus, Theorem 8 is stronger than Theorem 13 in [4].

In the following, we also present an example to compare the condition in Theorem 8 in this paper and that in Theorem 13 in [4].

Example 10. When $\mathscr{K}=R_{+}^{2}, F(x)=M x$, and $G(x)=x$, the (2) reduces to the linear complementarity problem (LCP) of finding vector $x^{*} \in R^{n}$ such that

$$
F\left(x^{*}\right) \geq 0, \quad G\left(x^{*}\right) \geq 0, \quad F\left(x^{*}\right)^{\top} G\left(x^{*}\right)=0,
$$

where $M=\left(\begin{array}{cc}1 & -3 \\ 0 & 1\end{array}\right)$.

It is easy to see that the solution set of the LCP $X^{*}=\{0\}$. In fact,

$$
\begin{gathered}
X^{*}=\left\{x \in R^{2} \| x \geq 0, M x \geq 0, x^{\top} M x=0\right\} \\
=\left\{\left(x_{1}, x_{2}\right) \in R^{2} \mid x_{1} \geq 0, x_{2} \geq 0, x_{1} \geq 3 x_{2},\right. \\
\left.x_{1}=\frac{3-\sqrt{5}}{2} x_{2} \text { or } x_{1}=\frac{3+\sqrt{5}}{2} x_{2}\right\} \\
=\{0\} .
\end{gathered}
$$

Clearly, $M$ is a $P$ matrix [17]. Thus, there exists constant $\tau>0$ such that

$$
\max _{1 \leq i \leq 2}\left\{(x-y)_{i}(M x-M y)_{i}\right\} \geq \tau\|x-y\|^{2}
$$


For any $x(\epsilon):=(-\epsilon ; \epsilon), \epsilon \geq 0$. By Theorem 8 , with $\gamma=1$, $\delta=1, A=I, U=I, B=0$, and $V=0$ and letting $\varphi_{1}(x):=$ $\left\|[F(x)]_{-}\right\|+\left\|[G(x)]_{-}\right\|+\left\|\left[F(x)^{\top} G(x)\right]\right\|^{1 / 2}$, we can obtain

$$
\frac{\|x(\epsilon)-0\|}{\varphi_{1}(x(\epsilon))}=\frac{\sqrt{2} \epsilon}{4 \epsilon+\epsilon+\sqrt{5} \epsilon} \longrightarrow \frac{\sqrt{2}}{5+\sqrt{5}}
$$

as $\epsilon \rightarrow 0$. Thus, Theorem 8 provides a global error bound for this LCP.

However, letting $x=(1 ; 1)$, we note that $x^{\top} M x=-1<0$ which shows that $M$ is not positive definite, so the condition that $F$ is strongly monotone in Theorem 13 in [4] does not hold. Thus, the result of Theorem 13 in [4] fails in providing an error bound for this LCP.

Secondly, if $F$ is $\gamma$-strongly $G$-monotone and $G$ is strongly $\delta$-nonexpanding, then it is easy to verify that

$$
\begin{aligned}
& \langle F(x)-F(y), G(x)-G(y)\rangle \\
& \quad \geq c_{5}\|G(x)-G(y)\|^{1+\gamma} \\
& \quad \geq c_{5} \alpha^{1+\gamma}\|x-y\|^{(1+\gamma) \delta}, \quad \forall x, y \in R^{n},
\end{aligned}
$$

where $c_{5}>0$ is constant. Moreover, the conditions that both $F$ and $G$ are Hölder continuous (or both $F$ and $G$ are Lipschitz continuous) in Theorem 8 in this paper are removed. Thus, Theorem 8 is stronger than Theorem 2.5 in [5]. Furthermore, by Theorem 2.1 in [5], the GNCP can be reformulated as general variational inequalities problem, and the conditions in Theorem 8 are also weaker than those in Theorem 3.1 in [11], Theorem 2 in [12], Theorem 3.1 in [13], and Theorem 3.1 in [16], respectively.

In the end of this paper, we will consider a special case of GNCP which was discussed in [11].

When $\mathscr{K}=R_{+}^{m}$, the (2) reduces to the generalization of the classical nonlinear complementarity problem of finding vector $x^{*} \in R^{n}$ such that

$$
F\left(x^{*}\right) \geq 0, \quad G\left(x^{*}\right) \geq 0, \quad F\left(x^{*}\right)^{\top} G\left(x^{*}\right)=0 .
$$

Combining this with Theorem 8 , we can also immediately obtain the following conclusion.

Corollary 11. Suppose that the hypotheses of Theorem 8 hold. Then there exists constant $\rho_{2}>0$ such that

$$
\begin{aligned}
& \operatorname{dist}\left(x, X^{*}\right) \\
& \leq \rho_{2}\left\{\left\|[F(x)]_{-}\right\|+\left\|[G(x)]_{-}\right\|+\left\|\left[F(x)^{\top} G(x)\right]\right\|^{1 / 2}\right\}^{2 /(1+\gamma) \delta}, \\
& \forall x \in R^{n} .
\end{aligned}
$$

Remark 12. It is clear that if $F$ is $\gamma$-uniform $p$-function and $G$ is strongly $\delta$-nonexpanding, for any $x, y \in R^{n}$, then

$$
\begin{aligned}
\max _{1 \leq i \leq m}\left\{[F(x)-F(y)]_{i}[G(x)-G(y)]_{i}\right\} \\
\quad \geq c_{1}\|G(x)-G(y)\|^{1+\gamma} \geq c_{1} \alpha^{1+\gamma}\|x-y\|^{(1+\gamma) \delta},
\end{aligned}
$$

so the condition in Corollary 11 is largely extended than the condition that $F$ is a uniform $p$-function with respect to $G$ (i.e., $\gamma=1, \delta=1$ ) in Theorem 3.3 in [11]. Moreover, the conditions that both $F$ and $G$ are Lipschitz continuous in Theorem 3.3 in [11] are removed. Thus, Corollary 11 is stronger than Theorem 3.3 in [11].

\section{Conclusion}

In this paper, we established a global error bound on the generalized nonlinear complementarity problems over a polyhedral cone, which improves the result obtained for variational inequalities and the GNCP in $[4,5,11-13]$ by weakening the assumptions. Surely, we may use the error bound estimation to establish quick convergence rate of the methods for the GNCP under milder conditions. This is a topic for future research.

\section{Conflict of Interests}

The authors declare that there is no conflict of interests regarding the publication of this paper.

\section{Acknowledgments}

The authors wish to give their sincere thanks to the associated editor and two anonymous referees for their valuable suggestions and helpful comments which improve the presentation of the paper. This work was supported by the Natural Science Foundation of China (nos. 11171180, 11101303, 11171362, and 11271226), the Specialized Research Fund for the Doctoral Program of Chinese Higher Education (20113705110002, and 20120191110031), the Shandong Provincial Natural Science Foundation (ZR2010AL005), the Shandong Province Science and Technology Development Projects (2013GGA13034), the Domestic Visiting Scholar Project for the Outstanding Young Teacher of Shandong Province Universities (2013), and the Applied Mathematics Enhancement Program of Linyi University.

\section{References}

[1] M. A. Noor, "General variational inequalities," Applied Mathematics Letters, vol. 1, no. 2, pp. 119-122, 1988.

[2] Y. J. Wang, F. M. Ma, and J. Z. Zhang, "A nonsmooth L-M method for solving the generalized nonlinear complementarity problem over a polyhedral cone," Applied Mathematics and Optimization, vol. 52, no. 1, pp. 73-92, 2005.

[3] X. Z. Zhang, F. M. Ma, and Y. J. Wang, "A Newton-type algorithm for generalized linear complementarity problem over a polyhedral cone," Applied Mathematics and Computation, vol. 169, no. 1, pp. 388-401, 2005.

[4] H. C. Sun and Y. J. Wang, "A sharper global error bound for the generalized nonlinear complementarity problem over a polyhedral cone," Abstract and Applied Analysis, vol. 2013, Article ID 209340, 13 pages, 2013.

[5] H. C. Sun and Y. J. Wang, "Global error bound estimation for the generalized nonlinear complementarity problem over a closed 
convex cone," Journal of Applied Mathematics, vol. 2012, Article ID 245458, 11 pages, 2012.

[6] H. C. Sun, Y. J. Wang, and L. Q. Qi, "Global error bound for the generalized linear complementarity problem over a polyhedral cone," Journal of Optimization Theory and Applications, vol. 142, no. 2, pp. 417-429, 2009.

[7] H. C. Sun and Y. J. Wang, "Further discussion on the error bound for generalized linear complementarity problem over a polyhedral cone," Journal of Optimization Theory and Applications, vol. 159, no. 1, pp. 93-107, 2013.

[8] R. Andreani, A. Friedlander, and S. A. Santos, "On the resolution of the generalized nonlinear complementarity problem," SIAM Journal on Optimization, vol. 12, no. 2, pp. 303-321, 2002.

[9] F. Facchinei and J. S. Pang, Finite-Dimensional Variational Inequality and Complementarity Problems, Springer, New York, NY, USA, 2003.

[10] J. S. Pang, "Error bounds in mathematical programming," Mathematical Programming, vol. 79, no. 1-3, pp. 299-332, 1997.

[11] N. H. Xiu and J. Z. Zhang, "Global projection-type error bounds for general variational inequalities," Journal of Optimization Theory and Applications, vol. 112, no. 1, pp. 213-228, 2002.

[12] M. V. Solodov, "Convergence rate analysis of iteractive algorithms for solving variational inequality problems," Mathematical Programming, vol. 96, no. 3, pp. 513-528, 2003.

[13] J. S. Pang, "A posteriori error bounds for the linearlyconstrained variational inequality problem," Mathematics of Operations Research, vol. 12, no. 3, pp. 474-484, 1987.

[14] J. Y. Han, N. H. Xiu, and H. D. Qi, Theory and Algorithms for Nonlinear Complementarity Problem, Shanghai Science and Technology Press, Shanghai, China, 2006, (Chinese).

[15] A. J. Hoffman, "On approximate solutions of systems of linear inequalities," Journal of Research of the National Bureau of Standards, vol. 49, no. 4, pp. 263-265, 1952.

[16] M. A. Noor, "Merit functions for general variational inequalities," Journal of Mathematical Analysis and Applications, vol. 316, no. 2, pp. 736-752, 2006.

[17] R. W. Cottle, J. S. Pang, and R. E. Stone, The Linear Complementarity Problem, Academic Press, Boston, Mass, USA, 1992. 


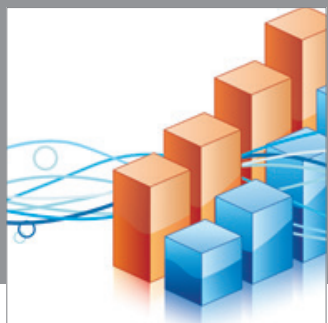

Advances in

Operations Research

mansans

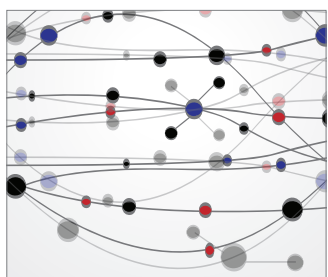

The Scientific World Journal
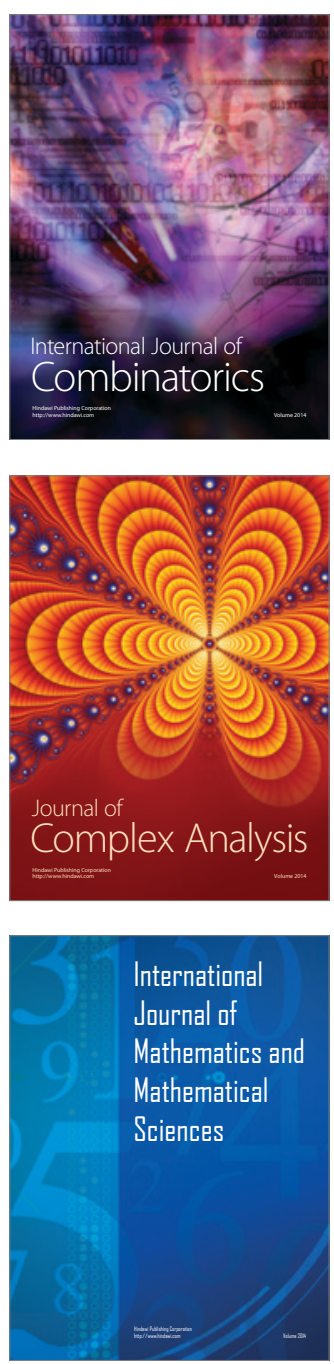
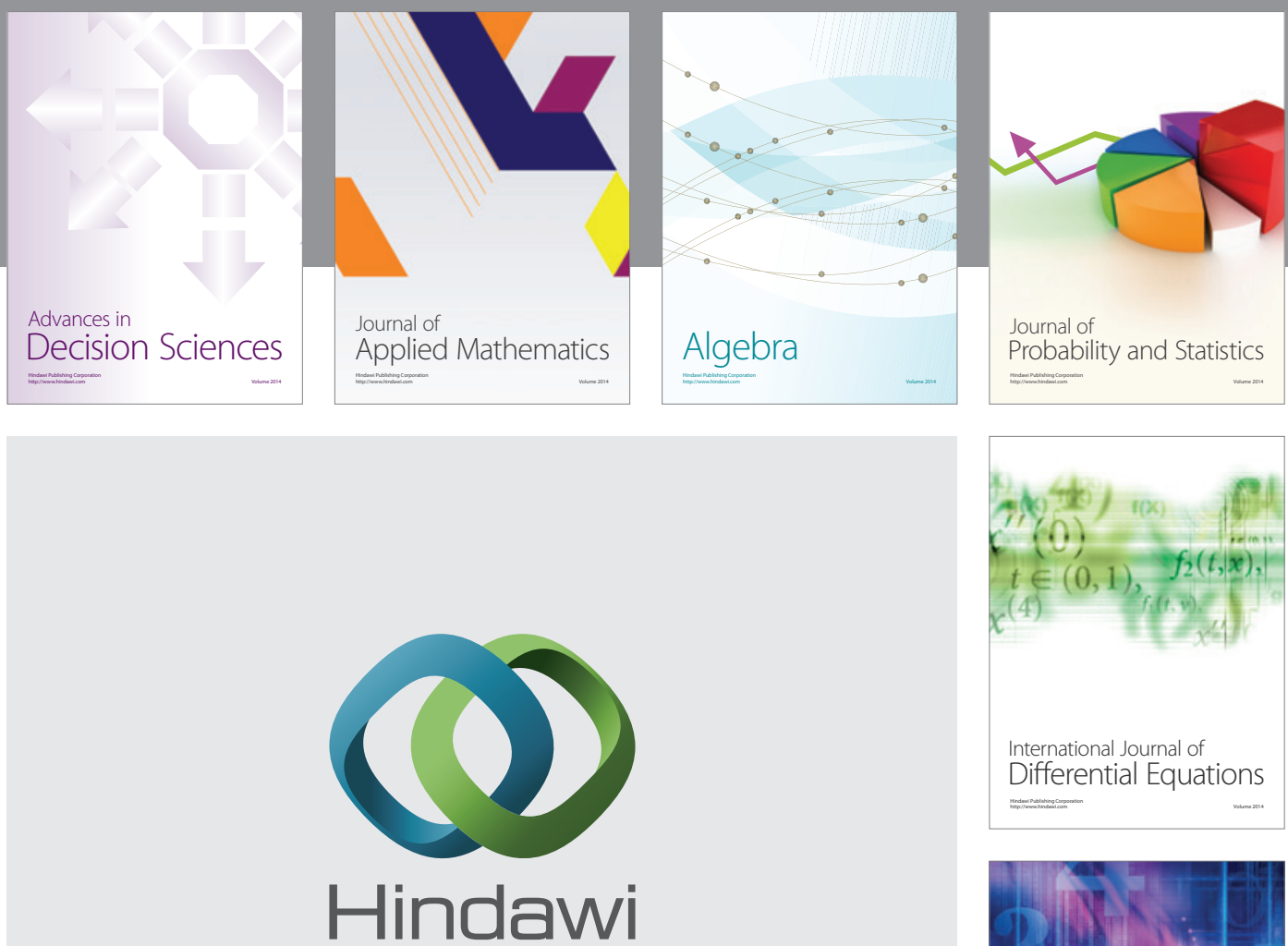

Submit your manuscripts at http://www.hindawi.com
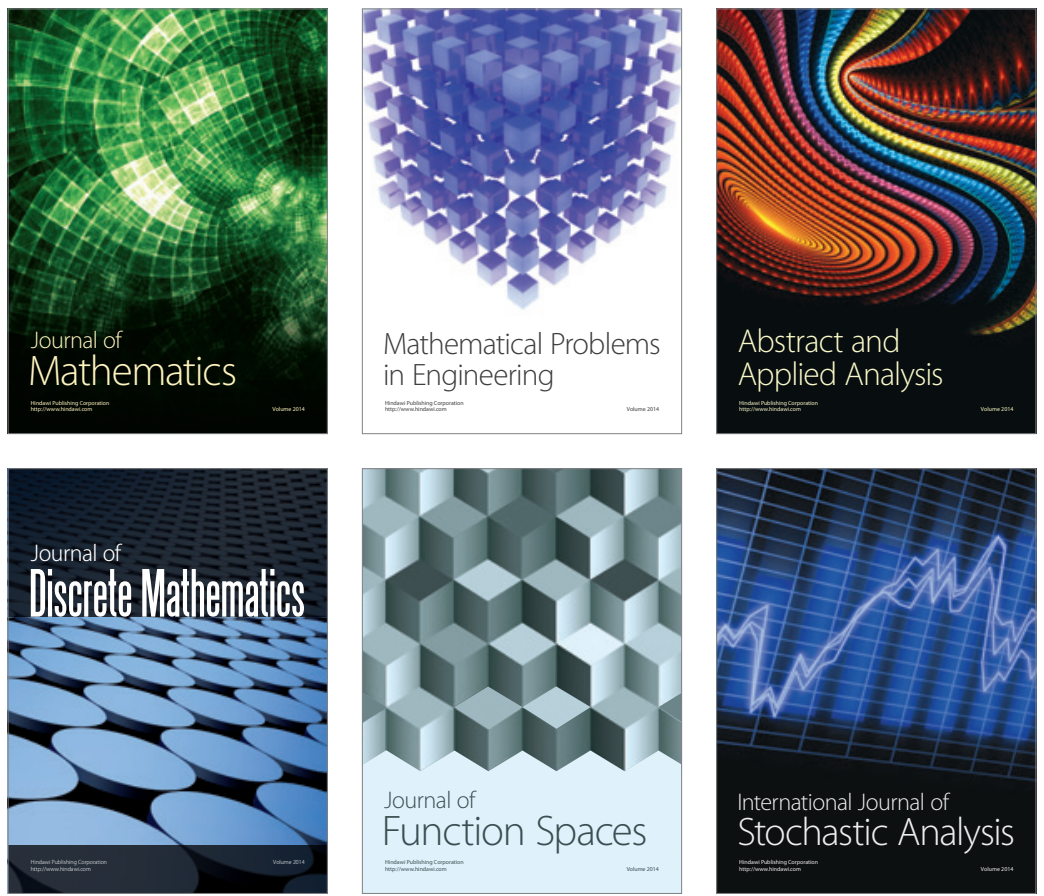

Journal of

Function Spaces

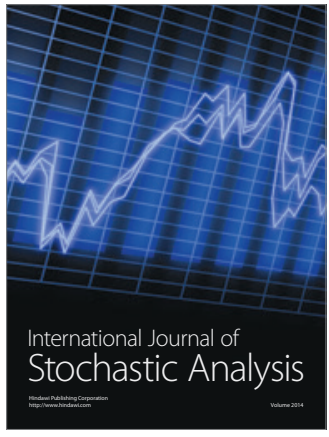

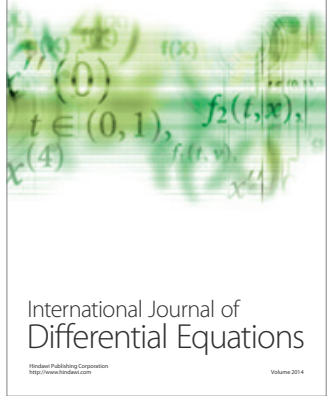
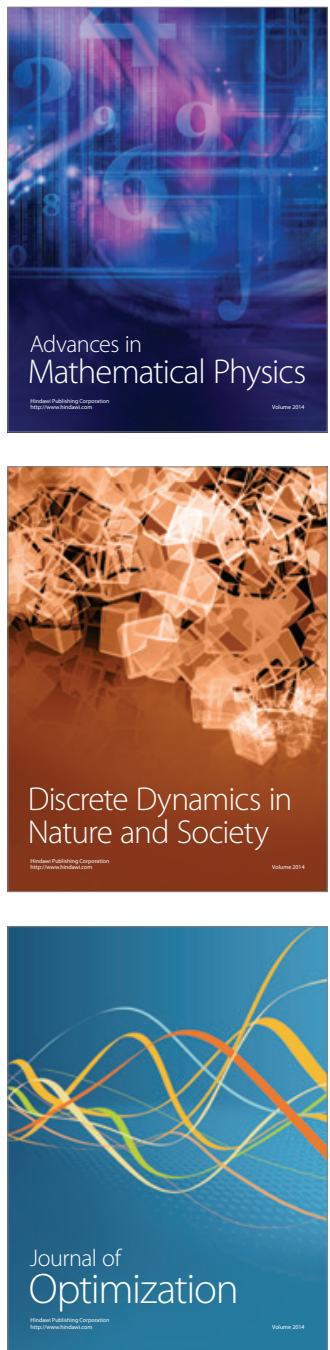